\title{
THE INTEGRAL OF THE SUPREMUM PROCESS OF BROWNIAN MOTION
}

\author{
SVANTE JANSON * AND \\ NICLAS PETERSSON,${ }^{* * *}$ Uppsala University
}

\begin{abstract}
In this paper we study the integral of the supremum process of standard Brownian motion. We present an explicit formula for the moments of the integral (or area) $\mathcal{A}(T)$ covered by the process in the time interval $[0, T]$. The Laplace transform of $\mathcal{A}(T)$ follows as a consequence. The main proof involves a double Laplace transform of $\mathcal{A}(T)$ and is based on excursion theory and local time for Brownian motion.
\end{abstract}

Keywords: Brownian motion; supremum process; local time; Brownian areas

2000 Mathematics Subject Classification: Primary 60J65

Secondary $60 \mathrm{~J} 55$

\section{Introduction}

Let $B(t), t \geq 0$, be a standard Brownian motion. Consider the following associated processes: the supremum process $S(t)=\max _{0 \leq s \leq t} B(s)$ and the local time $L(t)$, which can be regarded as a measure of the time $B(t)$ spends at 0 in the interval $[0, t]$; see [10, Chapter VI] for details. It is well known that these two processes, although pathwise quite different, have the same distribution [10, Chapter VI.2],

$$
\{S(t)\}_{t \geq 0} \stackrel{\mathrm{D}}{=}\{L(t)\}_{t \geq 0},
$$

where ‘ $\stackrel{\mathrm{D}}{=}$ denotes equality in distribution.

The purpose of this paper is to study the distribution of the area under $S(t)$ or, equivalently, $L(t)$ over a given time interval $[0, T]$. That is, the integral

$$
\mathcal{A}(T):=\int_{0}^{T} S(t) \mathrm{d} t \stackrel{\mathrm{D}}{=} \int_{0}^{T} L(t) \mathrm{d} t .
$$

For ease of notation, let $\mathcal{A}:=\mathcal{A}(1)$.

The area (1.1) appeared as a random parameter when analysing displacements for linear probing hashing. The Laplace transform of $\mathcal{A}$, which is presented in Corollary 2.1, provided the means to prove one of the main theorems in [9].

Note that the usual Brownian scaling

$$
\{B(T t)\}_{t \geq 0} \stackrel{\mathrm{D}}{=}\left\{T^{1 / 2} B(t)\right\}_{t \geq 0} \quad \text { for any } T>0,
$$

implies the corresponding scaling for the supremum process,

$$
\{S(T t)\}_{t \geq 0} \stackrel{\mathrm{D}}{=}\left\{T^{1 / 2} S(t)\right\}_{t \geq 0} .
$$

Received 18 July 2007; revision received 6 March 2009.

* Postal address: Department of Mathematics, Uppsala University, PO Box 480, SE-751 06 Uppsala, Sweden.

** Email address: niclasp@ math.uu.se 
Thus, for $T>0$,

$$
\mathcal{A}(T)=T \int_{0}^{1} S(T t) \mathrm{d} t \stackrel{\mathrm{D}}{=} T^{3 / 2} \mathcal{A},
$$

and it is enough to study $\mathcal{A}$.

\section{Results}

Let $\psi(s):=\mathrm{E}\left(\mathrm{e}^{-s \mathcal{A}}\right)$ denote the Laplace transform of $\mathcal{A}$. An essential part of this paper is devoted to proving the following formula for the Laplace transform of a variation of $\psi$, or in other words, a double Laplace transform of $\mathcal{A}$. Such formulae have already been derived for the integral of $|B(t)|$ and other similar integrals of processes related to Brownian motion; see [8] and the survey [3].

Theorem 2.1. Let $\psi$ be the Laplace transform of $\mathcal{A}$. For all $\alpha, \lambda>0$,

$$
\int_{0}^{\infty} \psi\left(\alpha s^{3 / 2}\right) \mathrm{e}^{-\lambda s} \mathrm{~d} s=\int_{0}^{\infty}\left(1+\frac{3 \alpha s}{2 \sqrt{2 \lambda}}\right)^{-2 / 3} \mathrm{e}^{-\lambda s} \mathrm{~d} s
$$

Remark 2.1. One of the parameters $\alpha$ and $\lambda$ in Theorem 2.1 can be eliminated (by setting it equal to 1 , for instance) without loss of generality. In fact, for any $\beta>0$, the formula is preserved by the substitutions $\lambda \mapsto \beta \lambda, \alpha \mapsto \beta^{3 / 2} \alpha$, and $s \mapsto \beta^{-1} s$.

The proof is given in Section 5. It is based on the excursion theory for Brownian motion and is inspired by similar arguments for other Brownian areas; see [8].

Theorem 2.2. The nth moment of $\mathcal{A}$ is

$$
\mathrm{E}\left(\mathcal{A}^{n}\right)=\frac{n ! \Gamma(n+2 / 3)}{\Gamma(2 / 3) \Gamma(3 n / 2+1)}\left(\frac{3 \sqrt{2}}{4}\right)^{n}, \quad n \in \mathbb{N} .
$$

Proof. Set $\lambda=1$ in Theorem 2.1 and denote the left- and right-hand sides by

$$
I(\alpha):=\int_{0}^{\infty} \psi\left(\alpha s^{3 / 2}\right) \mathrm{e}^{-s} \mathrm{~d} s
$$

and

$$
J(\alpha):=\int_{0}^{\infty}\left(1+\frac{3 \alpha s}{2 \sqrt{2}}\right)^{-2 / 3} \mathrm{e}^{-s} \mathrm{~d} s .
$$

The integrand of $I(\alpha)$ and all its derivatives with respect to $\alpha$ are dominated by functions of the form $s^{K} \mathrm{e}^{-s}$, uniformly in $\alpha>0$. Differentiation of $I(\alpha)$ is therefore allowed indefinitely due to dominated convergence. The same argument applies to $J(\alpha)$.

Also, the dominated convergence theorem shows that integration (with respect to $s$ ) can be interchanged with taking the limit $\alpha \rightarrow 0+$. Thus,

$$
\begin{aligned}
\lim _{\alpha \rightarrow 0+} \frac{\mathrm{d}^{n} I(\alpha)}{\mathrm{d} \alpha^{n}} & =\lim _{\alpha \rightarrow 0+} \int_{0}^{\infty} \frac{\mathrm{d}^{n}}{\mathrm{~d} \alpha^{n}} \psi\left(\alpha s^{3 / 2}\right) \mathrm{e}^{-s} \mathrm{~d} s \\
& =\int_{0}^{\infty} \lim _{\alpha \rightarrow 0+}\left(-s^{3 / 2}\right)^{n} \mathrm{E}\left(\mathcal{A}^{n} \exp \left\{-\alpha s^{3 / 2} \mathcal{A}\right\}\right) \mathrm{e}^{-s} \mathrm{~d} s \\
& =(-1)^{n} \mathrm{E}\left(\mathcal{A}^{n}\right) \int_{0}^{\infty} s^{3 n / 2} \mathrm{e}^{-s} \mathrm{~d} s \\
& =(-1)^{n} \Gamma\left(\frac{3 n}{2}+1\right) \mathrm{E}\left(\mathcal{A}^{n}\right)
\end{aligned}
$$


TABLE 1: The first four moments of $\mathcal{A}$.

\begin{tabular}{cc}
\hline$n$ & $\mathrm{E}\left(\mathscr{A}^{n}\right)$ \\
\hline 1 & $4 / 3 \sqrt{2 \pi}$ \\
2 & $\frac{5}{12}$ \\
3 & $64 / 63 \sqrt{2 \pi}$ \\
4 & $\frac{11}{24}$ \\
\hline
\end{tabular}

and

$$
\begin{aligned}
\lim _{\alpha \rightarrow 0+} \frac{\mathrm{d}^{n} J(\alpha)}{\mathrm{d} \alpha^{n}} & =\lim _{\alpha \rightarrow 0+} \int_{0}^{\infty} \frac{\mathrm{d}^{n}}{\mathrm{~d} \alpha^{n}}\left(1+\frac{3 \alpha s}{2 \sqrt{2}}\right)^{-2 / 3} \mathrm{e}^{-s} \mathrm{~d} s \\
& =\int_{0}^{\infty} \lim _{\alpha \rightarrow 0+} \frac{\Gamma(n+2 / 3)}{\Gamma(2 / 3)}\left(\frac{-3 s}{2 \sqrt{2}}\right)^{n}\left(1+\frac{3 \alpha s}{2 \sqrt{2}}\right)^{-n-2 / 3} \mathrm{e}^{-s} \mathrm{~d} s \\
& =\frac{\Gamma(n+2 / 3)}{\Gamma(2 / 3)}\left(\frac{-3}{2 \sqrt{2}}\right)^{n} \int_{0}^{\infty} s^{n} \mathrm{e}^{-s} \mathrm{~d} s \\
& =\frac{\Gamma(n+2 / 3)}{\Gamma(2 / 3)}\left(\frac{-3 \sqrt{2}}{4}\right)^{n} n !
\end{aligned}
$$

The fact that $I(\alpha)=J(\alpha)$ completes the proof.

The first four moments of $\mathcal{A}$ are listed in Table 1. Furthermore, Stirling's formula provides the asymptotic relation

$$
\mathrm{E}\left(\mathscr{A}^{n}\right) \sim \frac{2 \sqrt{3 \pi}}{3 \Gamma(2 / 3)} n^{1 / 6}\left(\frac{n}{3 \mathrm{e}}\right)^{n / 2}, \quad n \rightarrow \infty .
$$

Corollary 2.1. The Laplace transform of $\mathcal{A}$ is

$$
\psi(s)=\frac{1}{\Gamma(2 / 3)} \sum_{n=0}^{\infty} \frac{\Gamma(n+2 / 3)}{\Gamma(3 n / 2+1)}\left(\frac{-3 \sqrt{2} s}{4}\right)^{n} .
$$

Proof. The corollary follows from the identity

$$
\psi(s)=\sum_{n=0}^{\infty} \frac{(-s)^{n}}{n !} \mathrm{E}\left(\mathcal{A}^{n}\right) .
$$

Note that the sum converges absolutely for every complex $s$.

The graph of $\psi(s)$ is shown in Figure 1.

Remark 2.2. The Laplace transform of $\mathcal{A}$ can also be expressed in terms of generalised hypergeometric functions:

$$
\psi(s)={ }_{1} F_{1}\left(\frac{5}{6} ; \frac{4}{6} ; \frac{s^{2}}{6}\right)-\frac{4 s}{3 \sqrt{2 \pi}} 2 F_{2}\left(\frac{6}{6}, \frac{8}{6} ; \frac{7}{6}, \frac{9}{6} ; \frac{s^{2}}{6}\right) .
$$




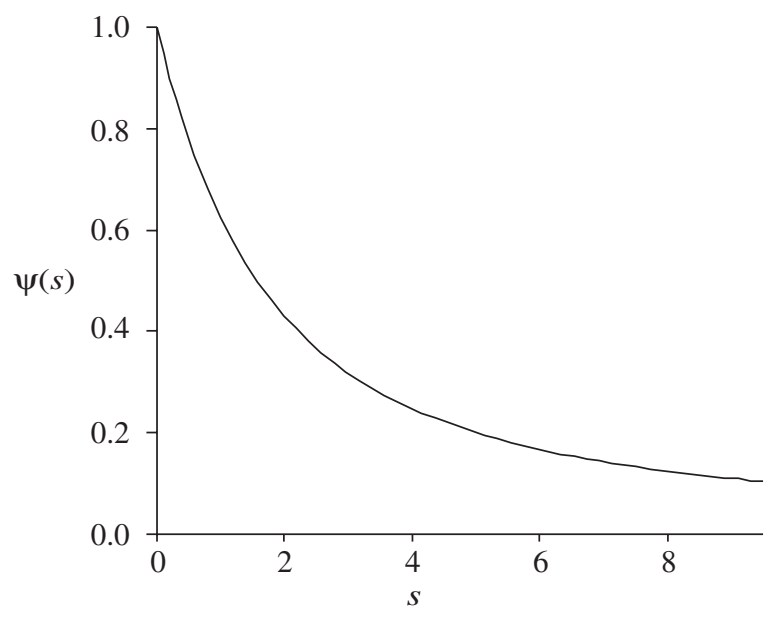

Figure 1: The Laplace transform of $\mathcal{A}$.

\section{Tail asymptotics}

Tauberian theorems by Davies [1] and Kasahara [7] (see [4, Theorem 4.5] for a convenient version) show that the moment asymptotics (2.1) imply the estimate $\ln \mathrm{P}(\mathcal{A}>x) \sim-3 x^{2} / 2$ for the tail of the distribution function. Thus, the following corollary is obtained.

Corollary 3.1. A has the tail estimate

$$
\mathrm{P}(\mathcal{A}>x)=\exp \left\{-\frac{3 x^{2}}{2}+o\left(x^{2}\right)\right\}, \quad x \rightarrow \infty .
$$

(This result can also be proved by large deviation theory; cf. similar results in [2].)

It seems difficult to obtain more precise tail asymptotics from the moment asymptotics, but it is natural to make a conjecture.

Conjecture 3.1. A has a density function $f_{\mathcal{A}}(x)$ satisfying

$$
f_{\mathcal{A}}(x) \sim \frac{2 \cdot 3^{1 / 6}}{\Gamma(2 / 3)} x^{1 / 3} \exp \left\{-\frac{3 x^{2}}{2}\right\}, \quad x \rightarrow \infty .
$$

In fact, if $\mathcal{A}$ has a density with $f_{\mathcal{A}}(x) \sim a x^{b} \exp \left\{-c x^{d}\right\}$ for some constants $a, b, c$, and $d$, then it is the only possible choice that yields the moment asymptotics (2.1); cf. [5].

Conjecture 3.1 may be compared with similar results for several Brownian areas in [5]; see also [3]. Note that in these results for Brownian areas, the exponent of $x$ is always an integer $(0,1$, or 2$)$. It is therefore of little surprise that here the exponent seems to be $\frac{1}{3}$, corresponding to the power $n^{1 / 6}$ in (2.1).

\section{Preliminaries on point processes}

Let $\mathfrak{S}$ be a measurable space. (In this paper, $\mathfrak{S}$ is either an interval of the real line or the product of two such intervals.) Although a point process $\Xi$ will be regarded as a random set $\left\{\xi_{i}\right\} \subset \mathfrak{S}$, it is technically convenient to formally define it as an integer-valued random 
measure $\sum_{i} \delta_{\xi_{i}}$. Hence, $\Xi(A)$ denotes the number of points $\xi_{i}$ that belong to a (measurable) subset $A \subseteq \mathfrak{S}$. Also, $x \in \Xi$ is equivalent to $\Xi(\{x\})>0$. For further details, see, e.g. [6].

A Poisson process with intensity $\mathrm{d} \mu$, where $\mathrm{d} \mu$ is a measure on $\mathfrak{S}$, is a point process $\Xi$ such that $\Xi(A)$ has a Poisson distribution with mean $\mu(A)$ for every measurable $A \subseteq \mathfrak{S}$ and $\Xi\left(A_{1}\right), \ldots, \Xi\left(A_{k}\right)$ are independent for every family $A_{1}, \ldots, A_{k}$ of disjoint measurable sets. Lemma 4.1, below, is a standard formula for Laplace functionals; see, e.g. [6, Lemma 12.2(i)].

Lemma 4.1. If $\Xi$ is a Poisson process with intensity $\mathrm{d} \mu$ on a set $\mathfrak{S}$ and $f: \mathfrak{S} \rightarrow[0, \infty)$ is a measurable function, then

$$
\mathrm{E}\left(\exp \left\{-\sum_{\xi \in \Xi} f(\xi)\right\}\right)=\exp \left\{-\int_{\mathfrak{S}}\left(1-\mathrm{e}^{-f(x)}\right) \mathrm{d} \mu(x)\right\} .
$$

Lemma 4.2, below, on the other hand, is more of a digression. The result follows from a standard gamma integral by integration by parts. (The result can also be written as $2 \Gamma\left(\frac{1}{2}\right) \lambda^{1 / 2}$.)

Lemma 4.2. If $\lambda>0$ then

$$
\int_{0}^{\infty}\left(1-\mathrm{e}^{-\lambda x}\right) x^{-3 / 2} \mathrm{~d} x=2 \sqrt{\pi \lambda}
$$

\section{Proof of Theorem 2.1}

The set $\{t: B(t)=0\}$ is almost surely (a.s.) closed and unbounded, so its complement $\{t: B(t) \neq 0\}$ is an infinite union of finite open intervals, denoted by $I_{v}=\left(g_{v}, d_{v}\right), v=$ $1,2, \ldots$, in some order. (The intervals cannot be ordered by appearance, since there is a.s. an infinite number of them in, say, [0,1]. Fortunately, the order does not matter.) The restrictions of $B(t)$ to these intervals are called the excursions of $B(t)$. Let $\hat{e}_{v}$ be the excursion during $I_{\nu}$.

The local time $L(t)$ is constant during each excursion. Let $\tau_{v}$ be the local time during $\hat{e}_{v}$ and let $\ell_{v}:=d_{v}-g_{v}$ be the length of $\hat{e}_{v}$. It is well known (see [10, Chapter XII]) that the collection of pairs $\left\{\left(\tau_{v}, \ell_{v}\right)\right\}_{\nu=1}^{\infty}$ forms a Poisson process in $[0, \infty) \times(0, \infty)$ with intensity

$$
\mathrm{d} \Lambda=\left(2 \pi \ell^{3}\right)^{-1 / 2} \mathrm{~d} \tau \mathrm{d} \ell .
$$

Note also that, a.s., if the excursion $\hat{e}_{\nu_{1}}$ comes before $\hat{e}_{\nu_{2}}$ then $\tau_{\nu_{1}}<\tau_{\nu_{2}}$.

Next, consider a Poisson process $\left\{T_{i}\right\}_{i=1}^{\infty}$ on $[0, \infty)$ with intensity $\lambda \mathrm{d} t$, independent of $\{B(t)\}$. Assume that the points are ordered with $0<T_{1}<T_{2}<\cdots$. Then $T_{1}, T_{2}-T_{1}, \ldots$ are i.i.d. $\operatorname{Exp}(\lambda)$ random variables with density function $\lambda \mathrm{e}^{-\lambda t}$. Furthermore, $T_{1}$ is independent of $\{B(t)\}$ and, thus, of $\{\mathcal{A}(T)\}$. It follows from (1.2) that $\mathcal{A}\left(T_{1}\right) \stackrel{\mathrm{D}}{=} T_{1}^{3 / 2} \mathcal{A}$ and, consequently,

$$
\mathrm{E}\left(\exp \left\{-\alpha \mathcal{A}\left(T_{1}\right)\right\}\right)=\mathrm{E}\left(\exp \left\{-\alpha T_{1}^{3 / 2} \mathcal{A}\right\}\right)=\mathrm{E}\left(\psi\left(\alpha T_{1}^{3 / 2}\right)\right)=\lambda \int_{0}^{\infty} \mathrm{e}^{-\lambda s} \psi\left(\alpha s^{3 / 2}\right) \mathrm{d} s
$$

The times $T_{i}$ are called marks, and an excursion is called marked if it contains at least one of the marks $T_{i}$. The marks $\left\{T_{i}\right\}$ are placed by first constructing $\{\mathrm{B}(\mathrm{t})\}$ and then adding marks according to independent Poisson processes with intensities $\lambda \mathrm{d} t$ in each excursion. Thus, given the excursions $\left\{\hat{e}_{v}\right\}$, each excursion $\hat{e}_{v}$ is marked with probability $1-\exp \left\{-\lambda \ell_{v}\right\}$, independently of the other excursions. The Poisson process $\Xi:=\left\{\left(\tau_{\nu}, \ell_{v}\right)\right\}$ defined by the excursions can be written as the union $\Xi^{\prime} \cup \Xi^{\prime \prime}$, where

$$
\Xi^{\prime}:=\left\{\left(\tau_{v}, \ell_{v}\right): \hat{e}_{v} \text { is unmarked }\right\}, \quad \Xi^{\prime \prime}:=\left\{\left(\tau_{v}, \ell_{v}\right): \hat{e}_{v} \text { is marked }\right\} .
$$


By the general independence properties of Poisson processes, $\Xi^{\prime}$ and $\Xi^{\prime \prime}$ are independent Poisson processes with intensities

$$
\mathrm{d} \Lambda^{\prime}:=\mathrm{e}^{-\lambda \ell} \mathrm{d} \Lambda=(2 \pi)^{-1 / 2} \ell^{-3 / 2} \mathrm{e}^{-\lambda \ell} \mathrm{d} \tau \mathrm{d} \ell
$$

and

$$
\mathrm{d} \Lambda^{\prime \prime}=\left(1-\mathrm{e}^{-\lambda \ell}\right) \mathrm{d} \Lambda=(2 \pi)^{-1 / 2} \ell^{-3 / 2}\left(1-\mathrm{e}^{-\lambda \ell}\right) \mathrm{d} \tau \mathrm{d} \ell
$$

respectively. In particular, if the lengths are ignored, the local times of the marked excursions form a Poisson process $\tilde{\Xi}$ on $(0, \infty)$ with intensity

$$
\int_{\ell=0}^{\infty}\left(1-\mathrm{e}^{-\lambda \ell}\right) \mathrm{d} \Lambda=\tilde{\lambda} \mathrm{d} \tau
$$

where, using Lemma 4.2,

$$
\tilde{\lambda}=\int_{0}^{\infty}(2 \pi)^{-1 / 2} \ell^{-3 / 2}\left(1-\mathrm{e}^{-\lambda \ell}\right) \mathrm{d} \ell=\sqrt{2 \lambda} .
$$

Owing to the fact that $B\left(T_{1}\right) \neq 0$ a.s., there exists a unique excursion $\hat{e}_{v^{*}}$ that contains the first mark $T_{1}$, i.e. $T_{1} \in I_{\nu^{*}}$. Let $\zeta:=L\left(T_{1}\right)=\tau_{v^{*}}$ be the local time at $T_{1}$ (and, thus, during $\hat{e}_{v^{*}}$ ). Since $\hat{e}_{v^{*}}$ is the first marked excursion, its local time $\zeta$ is the first of the points in the Poisson process $\tilde{\Xi}$ and, hence,

$$
\zeta \sim \operatorname{Exp}(\sqrt{2 \lambda})
$$

The restriction of $B(t)$ to the interval $\left[0, T_{1}\right]$ consists of all excursions $\hat{e}_{v}$ with local time $\tau_{\nu}<\tau_{v^{*}}=\zeta$ and the part of $\hat{e}_{\nu^{*}}$ on $\left(g_{v^{*}}, T_{1}\right)$, plus the set

$$
\left[0, T_{1}\right] \backslash \bigcup_{\nu} I_{\nu}=\left\{t \leq T_{1}: B(t)=0\right\},
$$

which, a.s., has measure 0 and, thus, may be ignored. Consequently, since $L(t)=\tau_{v}$ on $I_{\nu}$,

$$
\begin{aligned}
\mathcal{A}\left(T_{1}\right) & :=\int_{0}^{T_{1}} L(t) \mathrm{d} t \\
& =\sum_{\nu: \tau_{v}<\tau_{v^{*}}} \int_{I_{v}} L(t) \mathrm{d} t+\int_{g_{v^{*}}}^{T_{1}} L(t) \mathrm{d} t \\
& =\sum_{\nu: \tau_{v}<\zeta} \tau_{\nu} \ell_{v}+\zeta\left(T_{1}-g_{v^{*}}\right) \\
& =: \mathcal{A}^{\prime}+\mathcal{A}^{\prime \prime} .
\end{aligned}
$$
Thus,

The sum defined as $\mathscr{A}^{\prime}=\sum_{v: \tau_{v}<\zeta} \tau_{\nu} \ell_{\nu}$ only contains terms for unmarked excursions $\hat{e}_{\nu}$.

$$
\mathcal{A}^{\prime}=\sum_{\left(\tau_{\nu}, \ell_{\nu}\right) \in \Xi^{\prime}: \tau_{\nu}<\zeta} \tau_{\nu} \ell_{\nu} .
$$

Recall that $\zeta$ is determined by $\Xi^{\prime \prime}$ (as the smallest $\tau$ with $(\tau, \ell) \in \Xi^{\prime \prime}$ for some $\ell$ ) and that $\Xi^{\prime}$ and $\Xi^{\prime \prime}$ are independent. Hence, $\Xi^{\prime}$ and $\zeta$ are independent. It follows from Lemma 4.1, with $\mathfrak{S}=(0, \zeta) \times(0, \infty)$ and $f((\tau, \ell))=\alpha \tau \ell$, that

$$
\mathrm{E}\left(\mathrm{e}^{-\alpha \mathcal{A}^{\prime}} \mid \zeta\right)=\exp \left\{-\int_{\tau=0}^{\zeta} \int_{\ell=0}^{\infty}\left(1-\mathrm{e}^{-\alpha \tau \ell}\right) \mathrm{d} \Lambda^{\prime}(\tau, \ell)\right\} .
$$


By (5.2) and Lemma 4.2,

$$
\begin{aligned}
\int_{\tau=0}^{\zeta} \int_{\ell=0}^{\infty}\left(1-\mathrm{e}^{-\alpha \tau \ell}\right) \mathrm{d} \Lambda^{\prime}(\tau, \ell) & =\int_{\tau=0}^{\zeta} \int_{\ell=0}^{\infty}\left(1-\mathrm{e}^{-\alpha \tau \ell}\right)(2 \pi)^{-1 / 2} \ell^{-3 / 2} \mathrm{e}^{-\lambda \ell} \mathrm{d} \ell \mathrm{d} \tau \\
& =(2 \pi)^{-1 / 2} \int_{\tau=0}^{\zeta} \int_{\ell=0}^{\infty}\left(\mathrm{e}^{-\lambda \ell}-\mathrm{e}^{-(\lambda+\alpha \tau) \ell}\right) \ell^{-3 / 2} \mathrm{~d} \ell \mathrm{d} \tau \\
& =\int_{\tau=0}^{\zeta} \sqrt{2}(\sqrt{\lambda+\alpha \tau}-\sqrt{\lambda}) \mathrm{d} \tau \\
& =\frac{2 \sqrt{2}}{3 \alpha}\left((\lambda+\alpha \zeta)^{3 / 2}-\lambda^{3 / 2}\right)-\sqrt{2 \lambda} \zeta,
\end{aligned}
$$

and it follows that

$$
\mathrm{E}\left(\mathrm{e}^{-\alpha \mathcal{A}^{\prime}} \mid \zeta\right)=\exp \left\{\sqrt{2 \lambda} \zeta-\frac{2 \sqrt{2}}{3 \alpha}\left((\lambda+\alpha \zeta)^{3 / 2}-\lambda^{3 / 2}\right)\right\}
$$

Now consider $\mathcal{A}^{\prime \prime}=\zeta\left(T_{1}-g_{\nu^{*}}\right)$. Note that $T_{1}-g_{\nu^{*}}$ is the location (relative to the left endpoint of the excursion) of the first mark in the first marked excursion. Since $\Xi$ is a Poisson process with intensity independent of $\tau$, the location $T_{1}-g_{v^{*}}$ is independent of the local time $\zeta$ of the first marked excursion. Furthermore, the joint distribution of $\left(\ell_{v^{*}}, T_{1}-g_{v^{*}}\right)$ has density

$$
(\tilde{\lambda})^{-1} \lambda \mathrm{e}^{-\lambda y}(2 \pi)^{-1 / 2} \ell^{-3 / 2} \mathrm{~d} \ell \mathrm{d} y, \quad 0<y<\ell<\infty,
$$

where the normalisation constant $\tilde{\lambda}$ is given by (5.3). Consequently,

$$
\begin{aligned}
\mathrm{E}\left(\mathrm{e}^{-\alpha \mathcal{A}^{\prime \prime}} \mid \zeta\right) & =\mathrm{E}\left(\exp \left\{-\alpha \zeta\left(T_{1}-g_{\nu^{*}}\right)\right\} \mid \zeta\right) \\
& =\int_{y=0}^{\infty} \int_{\ell=y}^{\infty} \mathrm{e}^{-\alpha \zeta y}(\tilde{\lambda})^{-1} \lambda \mathrm{e}^{-\lambda y}(2 \pi)^{-1 / 2} \ell^{-3 / 2} \mathrm{~d} \ell \mathrm{d} y \\
& =\pi^{-1 / 2} \lambda^{1 / 2} \int_{y=0}^{\infty} \mathrm{e}^{-(\lambda+\alpha \zeta) y} y^{-1 / 2} \mathrm{~d} y \\
& =\lambda^{1 / 2}(\lambda+\alpha \zeta)^{-1 / 2}
\end{aligned}
$$

Again, since $\Xi^{\prime}$ and $\Xi^{\prime \prime}$ are independent, $\mathcal{A}^{\prime}$ and $\mathcal{A}^{\prime \prime}$ are conditionally independent given $\zeta$. Thus, (5.5) and (5.6) yield

$$
\begin{aligned}
\mathrm{E}\left(\exp \left\{-\alpha \mathcal{A}\left(T_{1}\right)\right\} \mid \zeta\right) & =\mathrm{E}\left(\mathrm{e}^{-\alpha \mathcal{A}^{\prime}} \mid \zeta\right) \mathrm{E}\left(\mathrm{e}^{-\alpha \mathcal{A}^{\prime \prime}} \mid \zeta\right) \\
& =\left(\frac{\lambda}{\lambda+\alpha \zeta}\right)^{1 / 2} \exp \left\{\sqrt{2 \lambda \zeta}-\frac{2 \sqrt{2}}{3 \alpha}\left((\lambda+\alpha \zeta)^{3 / 2}-\lambda^{3 / 2}\right)\right\} .
\end{aligned}
$$

By (5.4), $\zeta$ has the density $\sqrt{2 \lambda} \mathrm{e}^{-\sqrt{2 \lambda} x}, x>0$, and it follows that

$$
\mathrm{E}\left(\exp \left\{-\alpha \mathcal{A}\left(T_{1}\right)\right\}\right)=\lambda \sqrt{2} \int_{0}^{\infty}(\lambda+\alpha x)^{-1 / 2} \exp \left\{-\frac{2 \sqrt{2}}{3 \alpha}\left((\lambda+\alpha x)^{3 / 2}-\lambda^{3 / 2}\right)\right\} \mathrm{d} x .
$$

Finally, the substitution

$$
\frac{2 \sqrt{2}}{3 \alpha \lambda}\left((\lambda+\alpha x)^{3 / 2}-\lambda^{3 / 2}\right) \mapsto s
$$


provides the slightly simpler formula

$$
\mathrm{E}\left(\exp \left\{-\alpha \mathcal{A}\left(T_{1}\right)\right\}\right)=\lambda \int_{0}^{\infty}\left(1+\frac{3 \alpha s}{2 \sqrt{2 \lambda}}\right)^{-2 / 3} \mathrm{e}^{-\lambda s} \mathrm{~d} s .
$$

The result now follows by a comparison with (5.1).

\section{References}

[1] DAviEs, L. (1976). Tail probabilities for positive random variables with entire characteristic functions of very regular growth. Z. Angew. Math. Mech. 56, 334-336.

[2] Fill, J. A. And Janson, S. (2009). Precise logarithmic asymptotics for the right tails of some limit random variables for random trees. Ann. Combinatorics 12, 403-416.

[3] JANSON, S. (2007). Brownian excursion area, Wright's constants in graph enumeration, and other Brownian areas. Prob. Surveys 4, 80-145.

[4] Janson, S. And Chassaing, P. (2004). The center of mass of the ISE and the Wiener index of trees. Electron. Commun. Prob. 9, 178-187.

[5] Janson, S. And Louchard, G. (2007). Tail estimates for the Brownian excursion area and other Brownian areas. Electron. J. Prob. 12, 1600-1632.

[6] Kallenberg, O. (2002). Foundations of Modern Probability, 2nd edn. Springer, New York.

[7] Kasahara, Y. (1978). Tauberian theorems of exponential type. J. Math. Kyoto Univ. 18, 209-219.

[8] Perman, M. and Wellner, J. A. (1996). On the distribution of Brownian areas. Ann. Appl. Prob. 6, 1091-1111.

[9] Petersson, N. (2008). The maximum displacement for linear probing hashing. Preprint, U.U.D.M. Rep. 2008:6, Uppsala University.

[10] Revuz, D. ANd Yor, M. (1999). Continuous Martingales and Brownian Motion, 3rd edn. Springer, Berlin. 